\title{
EDUCACIÓN Y DIVERSIDAD: LA \\ CUESTIÓN DEL OTRO, SU IDENTIDAD Y NARRATIVA EN EL TIEMPO
}

\section{EDUCATION AND DIVERSITY: THE QUESTION OF THE OTHER, HIS IDENTITY AND NARRATIVE IN TIME}

DORYS ORTIZ G. ${ }^{1}$

Recibido: 29 de mayo de 2018

Aceptado: 22 de octubre de 2018

\footnotetext{
${ }^{1}$ Pontificia Universidad Católica del Ecuador, Facultad de Psicología, Quito, Ecuador (dortiz107@puce.edu.ec)
} 
ill|lil 52 


\section{EDUCACIÓN Y DIVERSIDAD: LA \\ CUESTIÓN DEL OTRO, SU IDENTIDAD \\ Y NARRATIVA EN EL TIEMPO}

\section{EDUCATION AND DIVERSITY: THE QUESTION OF THE OTHER, HIS IDENTITY AND NARRATIVE IN TIME}

Dorys Ortiz G.

Palabras Clave: el otro, identidad, narrativa, tiempo, educación, diversidad

Keywords: the other, identity, narrative, time, education, diversity

\section{RESUMEN}

El artículo plantea una reflexión acerca de la educación y la diversidad desde dos perspectivas: una psicológica y otra filosófica. El objetivo es esbozar el fundamento de la diversidad y cómo se debe pensar una educación diversa. Estos aspectos son de suma importancia en el mundo actual ya que se pretende homogeneizar a los individuos y promover un sentido de individualismo que di- ficulta una mejor comprensión del otro. Como hipótesis central se plantea que lo esencial en la diversidad es el otro, su identidad y la narrativa que se extiende en el tiempo y que tiene derivaciones en la educación. La reflexión se realiza con una metodología analítica, para la cual se ha dividido el texto en tres segmentos: la cuestión del otro, su identidad y narrativa, la educación, y la diversidad. 


\begin{abstract}
This article proposes a reflection on education and diversity from a psychological and philosophical perspective. The objective is to outline the foundation of diversity and how it should be sustained in a diverse education. These aspects are of great importance in today's world, which aims to homogenize individuals, as well as promoting a sense of individualism that makes it diffi-

central hypothesis, it is argued that the essential diversity is the question of the other, their identity and narrative, which extends over time and has derivations in education. The reflection is made using an analytical methodology for which the text has been divided into three segments: the question of the other, the other's identity and narrative, and education and diversity.
\end{abstract} cult to better understand the other. As a

\title{
INTRODUCCIÓN
}

El presente trabajo plantea una reflexión acerca de la educación y la diversidad desde dos perspectivas: una psicológica y otra filosófica. Se lo propone, de esta forma, porque la diversidad plantea una cuestión fundamental acerca de la identidad de las personas, la cual se vincula con su ser, ámbito bordado desde estas dos perspectivas para comprenderse mejor.

El objetivo de este artículo es plantear el fundamento de la diversidad -que es el otro- y esbozar cómo esta perspectiva debería pensarse en una educación diversa, no tanto por su variedad, sino esencialmente por su interés en la formación de las personas.
El mundo actual se debate entre dos tendencias opuestas: por un lado, existe una fuerte presión por parte de ciertas instituciones para la homogeneización de procesos, aspecto que se generaliza también a las personas y favorece algún grado de ceguera para sus características particulares; y, por otro lado, se promueve un amplio sentido de individualismo, en el cual predomina el sujeto con sus propias características sin tomar en cuenta al otro.

Estas dos tendencias dificultan el logro de una mejor comprensión del otro, quien es olvidado en el proceso. En la homogeneización cada uno se pierde como individuo en el grupo y en la 
individualización. En cambio, el sujeto predomina en tanto que borra completamente a las demás personas. Así, estos dos polos yo-otro deben sintetizarse en una comprensión de la alteridad inherente a las relaciones humanas.

La implicación se plantea el volver a las bases mismas de la Educación, cuyo interés primordial y ético debe estar en la formación del otro, el cual aparece diverso y diferente. En consecuencia, se tiende a poseer su forma particular de asumir y enfrentar la vida. Así, entender la tensión yo/otro desde una perspectiva psicológica y filosófica es un tema de actualidad, ya que permite plantear el sustento adecuado para el trabajo educativo desde una mirada diversa.
Se plantea como hipótesis básica de esta reflexión que lo esencial en la diversidad es la cuestión del otro, su identidad y la narrativa que se extiende en el tiempo y que tiene derivaciones en la educación. La metodología usada es analítica y reflexiva en vista de las disciplinas escogidas.

El texto se divide en tres grandes acápites. El primero aborda la cuestión del otro desde una perspectiva fenomenológica y psicológica; el segundo aborda la cuestión de la identidad y la narrativa y cómo se desenvuelven en el tiempo; para terminar, el tercero reflexiona acerca de la educación y cómo esta debería pensarse tomando en consideración los planteamientos hechos.

\section{LA CUESTIÓN DEL OTRO}

Toda la idea de la compasión se basa en la aguda conciencia de la interdependencia de todos los seres vivos que son parte uno del otro y todos están involucrados el uno en el otro.

Thomas Merton

Para comprender esta cuestión, filosóficamente, es necesario remitirse a la fenomenología, siguiendo los planteamientos de Merleau-Ponty (1994). Tres razones orientan esta opción: la primera de ellas es que, en la fenomenología de este autor, la percepción tiene una función primordial. Se la considera un proceso activo ya que permite una apertura a las características de la vida y, en esto, 
es posible reconocer algo del trabajo de Heidegger, tan preciado para la autora del presente texto.

La segunda razón es que Merleau-Ponty (1994) coincide con Husserl en las implicaciones acerca de la actividad de la conciencia puesto que en ellas existe un ser humano que tiene una conciencia, la cual siempre percibe algo. Se trata de una conciencia intencional, y como tal, re-afirma una noción concreta basada en lo real de las capacidades del ser humano.

La última razón, según lo que se ha comprendido de la postura de Merleau-Ponty (1994), es que la conciencia intencional puesto que permite la percepción de algo (el otro) establece una relación existencial y genera un compromiso por el cual, cada uno se ve (o debería verse) impulsado a reconocer a ese otro como semejante en tanto que sujeto y no, como un objeto percibido por la conciencia.

Entonces, volviendo a la fenomenología, Merleau-Ponty (1994) afirma que se trata "del estudio de las esencias, las cuales se sitúan en la existencia humana, con lo que se puede comprender al ser humano y al mundo en su "facticidad" (p. 7), es decir, que ambos tienen una existencia corpórea, física, previa a todo pensamiento o reflexión. Entonces, se considera al otro como un hecho concreto, con una existencia específica, particular y diversa.

Para estudiar las esencias se usa el método fenomenológico basado en la descripción que emplea, en primer lugar, la captación de los fenómenos (Merleau-Ponty, 1994): "Todo cuanto sé del mundo, incluso lo sabido por ciencia, lo sé a partir de una visión más o de una experiencia del mundo sin la cual, nada significarían los símbolos de la ciencia" (p. 8).

Se trata de un encuentro posible entre el propio ser y el del otro con características propias que los distinguen y que constituyen su identidad particular que se expresa en forma diversa, aspecto que se percibe mejor en el contexto educativo. Sin embargo, la diversidad no implica solo las diferencias ni se reduce a ellas. Desde el ámbito psicológico, se involucran ciertas características y la captación de las mismas, lo cual se realiza gracias a la conciencia.

La conciencia facilita el trabajo al ordenar, agrupar y clasificar las cosas, por lo que volver a las formas básicas y esenciales del mundo (el ser humano incluido en él) implica retornar a la naturaleza antes del conocimiento. Así, la diversidad se capta cuando se experimenta el mundo y cada persona en él, de manera reflexiva y consciente, razón por la cual, se parte de la idea de que (Merleau-Pon- 
ty, 1994): "Toda consciencia es consciencia de algo" (p. 17)'. Existe un ser humano que la posee y gracias a ella accede a sí mismo y al otro.

La conciencia permite acceder a lo que son las cosas y también a la forma en que han sido denominadas, antes de todo retorno a uno mismo, según Merleau-Ponty (1994): "El mundo no es lo que yo pienso, sino lo que yo vivo; estoy abierto al mundo, comunico indudablemente con él, pero no lo poseo; es inagotable" (p. 16).

Entonces, "tener conciencia es estar en relación con lo que es" (Lévinas, 1977, p. 183), es tener la capacidad de asumirse uno mismo y mantener una relación con el otro. Lo que pasa por la percepción es el proceso psicológico por el que cada persona recibe la impresiones del mundo, de los demás y de sí mismo y las organiza en su mente (Gross, 2012).

Pero esta captación tiene matices y diferencias propias del desarrollo psicológico del individuo (Papalia, Wendkos, \& Duskin, 2005), puesto que un niño percibe diferente a un adulto. Así, la diversidad no es una cuestión individual: su percepción, aceptación o rechazo es un tema esencialmente relacional.

El ser humano "está en el mundo y es en el mundo que se conoce" (Merleau-Ponty, 1994, p. 11) por lo que la percepción no se limita a una síntesis en el nivel de los juicios o de los actos, sino que está llena de variaciones que se pueden situar en el mundo, es decir, la diversidad existe y se manifiesta mucho antes de que cada individuo tenga una mirada sobre dicho fenómeno. El otro diverso es previo a cualquier conciencia, percepción o representación que se tenga de él. Su existencia es anterior. Sin embargo, tiene relación con uno mismo cuando se aproxima y se lo percibe.

La diversidad, por lo tanto, se muestra en una tensión entre yo y el otro, cada cual con sus propias características y particularidades intentando entrar en relación (Lévinas, 2008). De esta manera, es posible percibir al otro e intentar comprenderlo en su forma particular de existir, con sus características personales.

Según Merleau-Ponty (1994), para entender la dinámica entre el yo y el otro en su diversidad, se requiere la existencia de una visión en tres niveles: de sí mismo, del otro y del otro sobre uno mismo. Esta experiencia solo es posible cuando se reconoce que cada uno experimenta su propia existencia en sí mismo, ante los demás.

Cada persona es un otro que no puede ser reducido a la simple percepción, aunque se capten sus características. Su esencia es irreductible y se pertenece a sí misma, ya que solo se percibe aquello que está al alcance de la propia

\footnotetext{
${ }^{1}$ La idea original es de Husserl y hace referencia a la intencionalidad de la conciencia.
} 
conciencia y que el otro deja trascender, razón por la cual, "es preciso pensar en la heteronomía del Otro en el Mismo, donde el Otro no domina al Mismo sino que le despierta y le desilusiona" (Lévinas, 2008, p. 171).

El sí mismo es definido, en cierta medida, por el otro que percibe sus características y las señala o reacciona a ellas, pero, a la vez, cada uno impulsa a que el sí mismo perciba ciertas cosas y tenga conciencia de ellas, en forma de características particulares de color, tamaño y constitución. Se trata de una representación que surge de la percepción pero que es construida por el sí mismo.

En esta situación, el yo no está opuesto al Otro; más bien lo integra como parte constituyente de sí mismo (Lévinas, 1977). La contraposición yootro se resuelve en una síntesis, en la que el otro se integra en el sí mismo (Guidano, 1999) y el otro es percibido como seren-el-mundo (Ponce, 2005):

... Como generador de una dimensión de realidad irreducible y única, lo que implica el aceptar e interrogarse sobre el mundo del otro. $Y$ es en este proceso de ponerse en el lugar del sí mismo del otro, por el cual yo sustento en mí las pretensiones del otro como llamada de una interioridad que pide ser comprendida, que yo me cuestiono a mí mismo, mi orden corriente, mi modo de vivir y sentir (p. 56).
La mejor expresión de ese otro (y de uno mismo cuando es otro para los demás) es el rostro (Sudar, 1981), el cual muestra diversas facetas propuestas por Lévinas (1977) como el pobre, la viuda, el huérfano y el extranjero, citados como ejemplos paradigmáticos de los rostros que, además, son diversos. Por supuesto, esto no implica que frente a estos rostros haya un incremento o disminución de la alteridad, que no es algo cuantificable. Se trata más bien de un reconocimiento que implica aceptar y validar el derecho de las personas de mostrarse tal cuales son, en sus condiciones diversas, puesto que "[s]olo es posible el encuentro y el reconocimiento del trascendente, por y a través del rostro del otro" (Sudar, 1981, p. 95).

Ese rostro se percibe con una determinada expresión con la que cada persona se presenta a sí misma (Navarro, 2008, p. 181). Un rostro inexpresivo impide el acercamiento $y$, en consecuencia, niega el acceso al otro. Esto ya se percibe desde la más tierna infancia, en la relación entre el bebé y su madre, ya que es a través del rostro y de sus expresiones que el niño accede al mundo materno y viceversa (Papalia, Wendkos, \& Duskin, 2005). Sin expresiones, el mundo estaría vacío de sentido y de emociones.

Así, el sí mismo accede a su identidad al tomar conciencia de que es diferente a aquel rostro que percibe y 
al mismo tiempo se da cuenta de la alteridad, al percibir un rostro diferente al propio que, además, tiene un cierto grado de expresividad. Este intercambio genera una nueva lógica, completamente diferente: "La relación de alteridad parte de la capacidad del «rostro», de la mera presencia del otro, de cuestionar los poderes y la autoridad de la conciencia del yo" (Navarro, 2008, p. 183).

El rostro, inicialmente expresivo en gestos y miradas, articula, además, el lenguaje verbal, lo que constituye un segundo nivel de expresión que apoya o refuta aquello que se transmitió inicialmente. Es por esto que se considera que la relación yo-otro es asimétrica y diacrónica (Sudar, 1981, p. 99). Asimétrica puesto que los rostros expresan diferentes elementos que se armonizan o perturban mutuamente; diacrónica ya que existe un intervalo de tiempo entre un rostro y el otro, aun cuando el semblante del bebé coincida en el momento actual con el de su madre, sin embargo, el de ella existió previamente.

En esta relación, el niño (y su madre) adquieren un cierto grado de conciencia personal que se pone en contacto con el otro, ya que la construcción y consolidación de la propia identidad es previa a este encuentro (Feldman, 2007) o como lo señala Merleau-Ponty (1994):
Nosotros tomamos nuestro destino en manos, nos convertimos en responsables de nuestra historia mediante la reflexión, pero también mediante una decisión en la que empeñamos nuestra vida; y en ambos casos se trata de un acto violento que se verifica ejerciéndose (p. 20).

En la relación yo/otro se construye la historia personal, en la que toda palabra y gesto, es decir, todas las expresiones tienen un significado y expresan cierta toma de posición. Así, se plantea el reto importante de asumir las diferentes perspectivas propuestas por los rostros humanos hasta llegar al eje mismo del significado existencial, lo que Husserl ha denominado como "génesis del sentido", ya que "[p]or estar en el mundo estamos condenados al sentido; y no podemos hacer nada, no podemos decir nada que no tome un nombre en la historia" (Merleau-Ponty, 1994, p. 19).

En consecuencia, en este proceso de encuentro y desencuentro, cada uno va consolidando su propia identidad relacional. El encuentro yo/otro facilita el reconocimiento y la alteridad y, al mismo tiempo, la separación yo/otro favorece la diferenciación y la trascendencia, ya que separarse es trascender, por lo que es necesario reflexionar acerca de la identidad. 


\title{
DE LA IDENTIDAD Y OTRAS NARRATIVAS EN EL TIEMPO
}

\author{
Y retomé los hilos de mi vida, \\ Pero esta vez de colores, \\ Para volver a tejerme
}

Pero esta vez....

mucho más fuerte.

Anónimo

Ricoeur (2006) señala que una persona se reconoce en un personaje tomado de una trama cualquiera. Tal apropiación asume varias formas, desde la clara y simple imitación, hasta otra más abstracta, incluyendo el rechazo e incluso obteniendo una "justa distancia respecto a los modelos de identificación y de su poder de seducción" (p. 111).

La ganancia de esta apropiación crítica (Ricoeur, 2006) sería el "[a]prender a 'contarse' que también es aprender a contarse de otra manera" (p. 111). Así, el sí mismo se narra de cierta forma para lo que construye una trama particular. Este proceso también puede extenderse a la vida en general y asumir que, un individuo cualquiera es un lector en la vida diaria puesto que la lee y/o la escribe como si de un libro se tratase.

La trama del texto no es lo que interesa aquí, aun cuando es muy importante ya que es una experiencia diferente vivir una epopeya, una tragedia o una comedia, una vida de terror o de ciencia ficción. Sin embargo, lo que prima es lo que el individuo hace con ella. En este proceso, el lector se identifica de cierta forma con tal o cual personaje: desde la virgen más pura hasta la bruja más temible o desde el caballero de noble armadura hasta el villano más cruel, todos los personajes de la literatura pueden desarrollarse y encontrarse en la trama de la vida cotidiana.

Para llegar a reconocerse con un personaje dado, el lector debe hacer un proceso de identificación-distinción que es la única forma de aprender (Bateson, 1972), para lo cual, cada persona identifica ciertos rasgos en sí misma, luego los asume como propios y elabora una construcción apropiada a la que se denominará identidad, que se expresa en una narrativa relacional particular y en un tiempo definido. Se amplían estos aspectos a continuación. 


\section{La cuestión de la identidad}

La construcción de identidad implica el triple desafío (y riesgo)

de confiar en uno mismo, en los otros y también en la sociedad

Zigmunt Bauman

La construcción del sí mismo solo es posible como resultado de la tensión yo/otro: si el otro es muy semejante a uno mismo no hay posibilidad de establecer una distinción y si es muy diferente, tampoco es posible la identificación, razón por la cual, se vuelve muy importante, la diferencia, que solo se reconoce en la diversidad.

Ricoeur (2006) propuso el término de identidad narrativa (p. 111) para referirse a la conformación de una identidad a través de "un nuevo enfoque del concepto de ipseidad, planteando la relación dialéctica entre la identidad inmutable del idem, del mismo, y la identidad cambiante del ipse del sí, considerada en su condición histórica" (p. 111 y 112). Se trata de la diferencia que se percibe en "el mismo"y "él mismo".

En calidad de idem, la identidad tiene elementos fijos que permiten reconocer a un individuo $y$, en su calidad de ipse cambia, es mutable conforme la persona avanza en su vida. La identidad como mismisidad está relacionada con algunos factores (Ricoeur, 2008), de los cuales, solo se mencionará a la continui- dad ininterrumpida puesto que implica un sentido de permanencia a lo largo de un eje temporal, es decir, cada persona se distingue a sí misma y diferencia los elementos a su alrededor como siendo los mismos pese al tiempo transcurrido.

Así, la discontinuidad que podría amenazar la identidad solo puede ser manejada por este sentido de permanencia en el tiempo (Ricoeur, 2008, p. 111), que se expresa a través del carácter, entendiéndose por tal (Ricoeur, 2008): "El conjunto de signos distintivos que permiten identificar de nuevo a un individuo humano como siendo el mis mo" (p. 113). Se trata de una categoría que posee una naturaleza inmutable, con una perspectiva finita, que no ha sido elegida pero que da acceso a los valores y al uso de los poderes personales (decir, hacer, contar y contarse).

El carácter se muestra en actividades diarias que se convierten en costumbres, las cuales, a su vez, configuran ciertas disposiciones que se vuelven rasgos, es decir, "signos distintivos por los que se reconocen a una persona" (Ricoeur, 2008, p. 117), gracias a los cuales se la identifica. Esto solo es posible en un contexto de relaciones significativas en el que un individuo puede destacar tal o cual rasgo, por lo que Guidano (1999) sostiene que:

El sí-mismo es un sistema que se construye evolutivamente, b) que 
en esa construcción es definitorio el patrón vincular temprano que la persona, en la niñez, haya tenido con sus cuidadores; c) que la importancia de ese patrón vincular, en la primera etapa de la vida, radica en que la recurrencia de cierto tipo de hechos afectivos en la relación entre el cuidador y el niño dispara en éte la 'organización de un dominio emocional individual' que es la base sobre la que se constituye, durante el resto de su vida, el sentido unitario y continuo del sí-mismo de cada individuo (p. 337).

De esta concepción se desprenden tres consecuencias básicas (Balbi, 2004): (a) cada persona tiene una estructura particular que la identifica y la define; (b) esta estructura tiene como base un componente afectivo-emocional; (c) para pensar en cualquier cambio, es necesario conocer la estructura básica pero también la construcción adicional que cada persona ha hecho de sí misma a lo largo de su ciclo vital.

Con estos rasgos particulares que identifican a un individuo como tal o cual persona, él empieza a construir una historia que luego narra a los demás. Es por ello que se habla de una narrativa relacional.

\section{La narrativa relacional}

Los detalles son la esencia de una buena narración... $Y$ de una vida bien planteada. César Fernández

El individuo que, en su trama cotidiana, se ha identificado con un personaje tal y, en consecuencia, ha hecho una distinción de las características propias y ajenas que lo identifican, empieza a narrar una historia, es decir, elabora una narrativa particular en la que él mismo es el protagonista central.

Así, cada individuo (se) cuenta una historia puesto que da a conocer las características que lo identifican y también los aconteceres de su existencia lo cual, a su vez, mantiene su identidad. Este aspecto sobre el sí mismo implica que cada uno se expresa en un mundo relacional (Linares, 1996).

Una narración se construye en base a lo que dijeron y narraron las personas alrededor de uno mismo, en los primeros años y luego, se comienza a contar una historia... para alguien más. La identidad así construida a lo largo del tiempo se elabora en una relación inicial particular cuyo vehículo esencial es el lenguaje (Balbi, 2004) que permite una distinción interesante entre la experiencia misma y su narración. 
Esta posibilidad de tomar distancia de la experiencia inmediata y, luego, de narrarla en una secuencia ordenada de hechos "generó en los humanos un aumento de la sensibilidad para registrar los detalles de la subjetividad propia y ajena e impulsó el desarrollo de un sentido diferenciado de identidad personal" (Balbi, 2004, p. 324).

Así, la narrativa de sí mismo y de los acontecimientos vividos favoreció una modificación de la conciencia de los acontecimientos, los cuales se fueron ordenando en una secuencia temporal, en la cual es posible distinguir un inicio, un intermedio y un final, que se convierten en una trama particular en la que la epopeya, el drama, la comedia, el terror y la ciencia ficción se mezclan a partes iguales y, lo que es aún más interesante, en ellas cada uno muestra rasgos de héroe, villano, payaso, monstruo o mago, también, en ocasiones, a partes iguales.

Esto facilitó "la organización de la información proveniente de modulaciones autorreferenciales (sensoriales, propioceptivas, emocionales y afectivas) en torno de un sentido personal unitario y continuo en el tiempo" (Balbi, 2004, p. 324), en íntima relación con los demás puesto que esta regulación es relacional, en el momento en que la narración es un relato hecho para alguien más. Los individuos no se desarrollan en un vacío relacional ya que, cada uno crece en una díada básica que, a su vez, regula su narratividad (Bronfenbrenner, 1987).

Esta regulación tiene como base el reconocimiento puesto que, como Ricoeur (2006) ya la sostuvo: "[e]n el reconocimiento mutuo termina el recorrido del reconocimiento de sí mismo" (p. 238). Su modelo más básico y fundante es el amor que abarca las relaciones afectivas que marcan al sujeto: familia, amigos, pareja, etc. Todas conforman la base y la fuente del reconocimiento (o de su ausencia), gracias al establecimiento de un vínculo cuyo modelo se encuentra en la primera relación del sujeto con su madre.

Además, las relaciones circulan entre dos polos (Bowen, 1991; Ricoeur, 2006): el de la cercanía y el de la distancia. Su aprendizaje inicia en la niñez y, en la adultez, proporciona dos beneficios principales: recuperar la capacidad de independencia y, otro más doloroso pero necesario al fin si cada uno desea estructurarse como ser humano, como es el de estar solo que, a la vez, aumenta la confianza en la permanencia del vínculo invisible que se ha creado y que mantiene a los miembros del grupo unidos a pesar de la distancia y de la separación.

Esta es la base primaria del reconocimiento mutuo puesto que la madre y el hijo se reconocen (o no lo hacen) como sujetos dignos de confianza, suficiente como para mantener el vínculo 
pese a la separación, relación que se sostiene en la construcción de una narrativa personal asumida, sostenida o cuestionada por las personas alrededor. Esta narrativa se desenvuelve a lo largo de un eje temporal que se extiende desde el pasado hacia el futuro y se manifiesta en el momento actual. Los seres humanos (se) cuentan historias a sí mismos y a los demás lo que sustenta su propia identidad y permite reconocer la de los demás.

\section{El tiempo en la narrativa individual}

No podemos matar el tiempo

Sin herir la eternidad.

Anónimo

El sujeto, cuya identidad se expresa en una narrativa particular y lo hace para un público definido, lo realiza en un tiempo característico. Su narrativa se extiende en un horizonte temporal que se denomina días, horas, años y que tiene la doble cualidad de ser medido, pretenciosamente atrapado por los segmentos que lo componen, pero a la vez, ser elusivo que se escapa y no deja de pasar.

El tiempo es una dimensión básica para organizar la experiencia y constituye un componente esencial al momento de construir la propia identidad (Guidano, 1999):

Si bien todos experimentamos la dirección irreversible del tiempo desde el pasado hacia el futuro como una dimensión temporal "objetiva", cada uno de nosotros siente esta irreversibilidad dentro de un tiempo "subjetivo", que corre paralelo a, y es percibido como entrelazado con, el orden temporal objetivo. Además, todo desarrollo del ciclo vital va acompañado por la aparición gradual de cambios en la experiencia subjetiva de esta irreversibilidad del tiempo... (p. 120).

Esta experiencia subjetiva, asociada al ciclo de vida, determina que la vivencia del tiempo sea diferente en cada edad y conforme una estructura narrativa particular que concede significado a cada persona de acuerdo a su tiempo específico, razón por la cual, el gran reto es mantener la identidad mientras cambian ciertos rasgos, en el esfuerzo de asignar un significado particular a cada vivencia en la propia línea temporal, contando(se) una historia en el camino.

La narración, que se inscribe en un eje temporal, es una característica particular de la experiencia humana, cuya integración contribuye a la organización y consolidación de una unidad organizada y coherente, como lo dice Balbi (2004):

Las estructuras narrativas organizan y dan sentido a nuestra experiencia, pero ésta contiene siempre matices 
afectivos, emocionales, sensoriales e imaginativos, que no están presentes en nuestra imagen y en nuestros relatos sobre nosotros mismos. En cada historia de vida narrada quedan excluidas porciones importantes de sentimientos efectivamente ocurridos; y esta exclusión atencional específica, como un aspecto característico de la coherencia interna de cada sistema personal, tiene lugar en función del mantenimiento de un sentido estable y continuo de la propia identidad narrativa (Guidano, 1999) (p. 326).

Por lo tanto, el tiempo es una variable esencial al momento de comprender la identidad: permite su desenvolvimiento a través de una narrativa especial, en la que cada persona se cuenta a sí misma, su propia vida y, a la vez, va tejiéndola, al anudar los diferentes hilos de su trama particular, que se combina con la de los demás. Tal vez, es posible imaginar cambios drásticos en una persona que surgen de experiencias diversas y que llevarían a considerar que son varias personas en una sola y misma vida. Sin embargo, existen aspectos nucleares que forman la identidad de la persona que resultan muy refractarios al cambio. Aunque cambien totalmente de rostro, su identidad permanecerá, más o menos, constante.

Estos elementos: identidad, narrativa y tiempo, confluyen sin duda alguna en la educación, que se convierte así en la matriz de una integración vital puesto que la identidad de unos y otros y sus narrativas particulares se van entretejiendo como un tapiz, en el cual predomina la diversidad.

\section{EDUCACIÓN Y DIVERSIDAD}

Hay personas que están unidas de por vida Por un hilo que llamamos casualidad Tejido por una araña juguetona A la que llamamos destino Anónimo

Cietamente, para educar hay que considerar condiciones psicológicas, co- menzando desde fenómenos psíquicos básicos como la atención, la percepción 
y la memoria hasta llegar a aspectos más complejos como el lenguaje, el pensamiento, la inteligencia, y las emociones. Muchos estudios se han realizado hasta el momento actual sobre estos diversos temas (Allueva, 2002; Bisquerra, 2012; Calero, 2009; Flavell, 1976) y han señalado la influencia de estos aspectos en el proceso de aprendizaje.

Algunos de ellos (Calero, 2009) ya dan cuenta de la importancia de tomar en cuenta las diferencias, por ejemplo, en los estilos de aprendizaje (Klimenko, 2009), los cuales señalan la gran diversidad que existe en las aulas a la que los docentes se enfrentan día a día. Sin embargo, el fundamento de todo esto es la consideración primordial del otro como tal, en su calidad de diferente y diverso.

Lévinas (1977) señaló apropiadamente que el rostro es la mejor expresión del otro. Incluso plantea rostros paradigmáticos con los cuales es posible que el docente se encuentre en su labor diaria. Así, la cuestión de la diversidad se vuelve más importante, puesto que se trata de considerar simplemente (aunque difícilmente) al otro como tal.

Se trata de otro que se expresa en un lenguaje particular gracias al cual, construye permanentemente su identidad que se expresa en narrativas particulares que se van conectando con las de los demás. En este proceso es muy significativo el reconocimiento como elemento básico para acercarse al otro y comprenderlo. Según Ricoeur (2008) su mejor expresión es el amor.

Tomando en consideración estos elementos es posible definir la educación en la diversidad como (Moya \& Gil, 2018):

Un proceso amplio y dinámico de construcción y reconstrucción de conocimiento que surge a partir de la interacción entre personas distintas en cuanto a valores, ideas, percepciones, intereses, capacidades, estilos cognitivos y de aprendizaje, etc., que favorece la construcción, consciente y autónoma, de formas personales de identidad y pensamiento, y que ofrece estrategias y procedimientos educativos (enseñanza/aprendizaje) diversificados y flexibles con la doble finalidad de dar respuesta a una realidad heterogénea y de contribuir a la mejora y el enriquecimiento de las condiciones y relaciones sociales y culturales (Jiménez y Vilá, 1999, 199) (p. 4).

Cuando se asume esta perspectiva se establece la posibilidad de considerar a los individuos diversos como parte de una sociedad, ella misma tan diversa como los individuos que la componen. Por lo tanto, la educación, entendida como la base misma de la sociedad y su mejor expresión, puede favorecer la 
construcción de individuos más respetuosos y reconocedores de la diversidad que los acompaña en las aulas. Así, se podría aspirar a la construcción de sociedades más democráticas (Moya \& Gil, 2018) en las que todas las voces se escuchen y en las que cada narrativa individual sea un hilo más de las narrativas colectivas, que se inscriben en el tiempo.

Por lo tanto, para lograr una educación diversa, se considera importante tomar en consideración algunos aspectos.

El primero -y más importante- es tomar conciencia de que el otro es tal, tan semejante en ciertos procesos básicos, como solo un ser humano puede parecerse a otro, pero a la vez, tan diferente en cuanto a pensamientos, ideas, creencias, religión, cultura, etc.

Tomar conciencia de este aspec- to conlleva inevitablemente el hecho de tratar de alcanzar una comprensión más integral de cada persona. Esta asunción conlleva el reto de la colaboración, es decir, que, luego de asumir y comprender la diversidad, cada persona sea capaz de dar la mano a otra para realizar un diálogo que facilite un encuentro de identidades y permita su despliegue (Moya \& Gil, 2018).

Además, la diversidad conlleva un gran desafío para los docentes puesto que implica un trabajo desde una perspectiva intercultural (Ortiz, 2015). Se trata de un profesional que, asumiendo el reto de trabajar con la diversidad, lo hace desde la comprensión y aceptación (Moya \& Gil, 2018), desarrollando actividades y recursos que brinden apoyo positivo a los estudiantes.

\section{CONCLUSIONES}

El estudio de la educación y la diversidad tiene su anclaje en cuestiones psicológicas y filosóficas. En este último ámbito, la fenomenología favorece el estudio de la esencia de los fenómenos, considerándolos como el resultado de un contacto directo sin pensamiento mediador: una invitación a experimentar el mundo en su más clara y transparente naturaleza.
Este acercamiento se realiza gracias a la conciencia que permite acceder al mundo, a las personas y sus características. Se trata de aprehenderlas y darse cuenta de que existen de forma diferente a uno mismo. Así, se captan las esencias gracias a la percepción que organiza el cúmulo de sensaciones asimiladas, en un todo organizado que tiene sentido particular para cada ser humano. 
Conciencia y percepción son esenciales para entrar en relación con el otro, el cual es captado en su facticidad y organicidad. El otro y sus características se revela de tal forma a la conciencia personal, en una relación de mutua influencia, puesto que se perciben solo ciertos aspectos y no la totalidad puesto que el otro, es mucho más que aquello que se percibe.

La mejor forma en que el otro se manifiesta es en el rostro y en la expresión. Ambos son la síntesis de sí mismo y del diferente, del ajeno, de aquel ser, al que solo se accede a través de la lógica construida en uno mismo, durante el propio desarrollo, ya que solo se mira al otro, a partir de uno mismo.

La identidad es una construcción particular que cada persona hace en función de las experiencias que vive. Incluye aquellos aspectos propios del sí mismo y también aquellos rasgos que se han asumido, fruto de identificaciones personales y significativas para cada persona. Esta identidad se despliega en una narrativa particular que fue elaborada por el aporte de las historias que rodearon al individuo y, posteriormente, cada persona (se) narra una historia particular fruto de su propio recorrido vital.

La narrativa se extiende en el tiempo y, en ocasiones, trasciende mucho más allá de una persona ya que la historia de un individuo se entreteje con los relatos de su propia comunidad de pertenencia. Así, cada ser humano, con su identidad propia y particular es un hilo más en la historia de toda la humanidad.

Finalmente, una educación que se defina como diversa debe asumir el reto de tomar conciencia de la diversidad que existe en el mundo, tener la capacidad de captar las diferencias y asumir las identidades particulares que se despliegan en el contexto educativo. No se trata de atender a cada uno particularmente, si no de respetarlo como tal, como un ser diverso, se trata de otro con el que se entra en relación y cuya narrativa se entreteje con la propia, para formar un tapiz cuyos colores se manifiestan en el presente pero cuyos hilos se extienden hacia el futuro. 


\section{BIBLIOGRAFÍA}

Allueva, P. (12 de Abril de 2002). Desarrollo de habilidades metacognitivas: programa de intervención. Recuperado el 25 de Agosto de 2015, de Conceptos básicos sobre metacognición: http://www. unizar.es/ice/images/stories/materiales/curso_24_2012/Concepto-de-Metacognici\%C3\%B3n-PAllueva.pdf

Balbi, J. (2004). La mente narrativa. Hacia una concepción post-racionalista de la identidad personal. Buenos Aires: Paidós.

Bateson, G. (1972). Pasos hacia una ecología de la mente. Una aproximación revolucionaria a la autocomprensión del hombre. Buenos Aires: Lohlé-Lumen.

Bisquerra, R. (2012). Educación emocional y bienestar. España: Wolters Kluwer.

Bowen, M. (1991). De la familia al individuo: la diferenciación del sí mismo en el sistema familiar. Barcelona: Paidós.

Bronfenbrenner, U. (1987). La ecología del desarrollo humano. Barcelona: Paidós.

Calero, M. (2009). Aprendizaje sin límites: Constructivismo. México D.F.: Alfaomega.
Feldman, R. (2007). Desarrollo psicológico a través de la vida. México: Pearson Education.

Flavell, J. (1976). Metacognitive aspects of problem solving. En L. Resnik, The nature of intelligence. (págs. 231-235). Hillsdale: Erlbaum.

Gross, R. (2012). Psicología: la ciencia de la mente y la conducta. México: El Manual Moderno.

Guidano, V. (1999). El modelo cognitivo post-racionalista. Hacia una reconceptualización teórica y crítica. Bilbao: Desclée de Brouwer.

Klimenko, O. (2009). La enseñanza de las estrategias cognitivas y metacognitivas como una vía de apoyo para el aprendizaje autónomo en los niños con déficit de atención sostenida. Revista Virtual Universidad Católica del Norte, 1-20.

Lévinas, E. (1977). Totalidad e infinito. Ensayo sobre la exterioridad. Salamanca: Sígueme.

Lévinas, E. (2008). Dios, la muerte y el tiempo. Madrid: Ediciones Cátedra.

Linares, J. (1996). Identidad y narrativa. La terapia familiar en la práctica clínica. Barcelona: Paidós.

Merleau-Ponty, M. (1994). Fenomenología de la percepción. Barcelona: 
Planeta - De Agostini S.A.

Moya, A., \& Gil, M. (10 de Mayo de 2018).

La Educación del futuro: educación en la diversidad. Obtenido de RÁBIDA.UHU.ES: http://rabida. uhu.es/dspace/bitstream/handle/10272/3439/b15759763.pdf

Navarro, O. (2008). El "rostro" del otro: una lectura de la ética de la alteridad de Emmanuel Lévinas. Contrastes. Revista Internacional de Filosofía, 177-194.

Ortiz, D. (2015). La Educación Intercultural: el desafío de la unidad en la diversidad. Sophia, 91-110.
Papalia, D., Wendkos, S., \&Duskin, R. (2005). Desarrollo Humano. México: McGraw-Hill Interamericana.

Ponce, Á. (2005). Construcción de la otredad en procesos afectivos. Una aproximación a la situación de violencia. Quito: UPS.

Ricoeur, P. (2006). Caminos del reconocimiento. Tres estudios. México: Fondo de Cultura Económica.

Ricoeur, P. (2008). Sí mismo como otro. México: Siglo XXI.

Sudar, P. (1981). El rostro del pobre. Buenos Aires: Patria Grande. 